\title{
Factors that Predict Clinical Benefit of EGFR TKI Therapy in Patients with EGFR Wild-Type Lung Adenocarcinoma
}

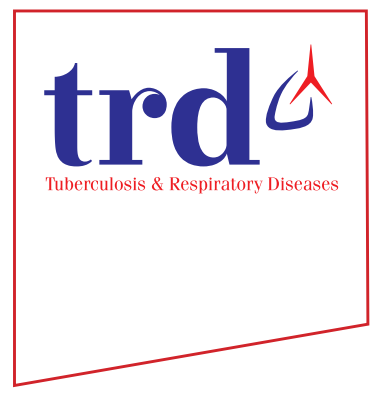

\author{
Seo Yun Kim, M.D. ${ }^{1, *}{ }^{\mathbb{D}}$, Jae Kyung Myung, M.D. ${ }^{2, *}$, Hye-Ryoun Kim, M.D. ${ }^{1}$, Im Il Na, M.D. Ph.D. ${ }^{3}$, \\ Jae Soo Koh, M.D., Ph.D. ${ }^{2}$, Hee Jong Baek, M.D., Ph.D. ${ }^{4}$ and Cheol Hyeon Kim, M.D., Ph.D. ${ }^{1}$ (iD \\ ${ }^{1}$ Division of Pulmonology, Department of Internal Medicine, ${ }^{2}$ Department of Pathology, ${ }^{3}$ Division of Hematology/Oncology, \\ Department of Internal Medicine, ${ }^{4}$ Department of Thoracic Surgery, Korea Cancer Center Hospital, Korea Institute of \\ Radiological and Medical Sciences, Seoul, Korea
}

Background: Epidermal growth factor receptor $(E G F R)$ mutations in non-small cell lung cancers have emerged as key predictive biomarkers in EGFR tyrosine kinase inhibitor (TKI) treatment. However, a few patients with wild-type EGFR also respond to EGFR TKIs. This study investigated the factors predicting successful EGFR TKI treatment in lung adenocarcinoma patients with wild-type EGFR.

Methods: We examined 66 patients diagnosed with lung adenocarcinoma carrying wide-type EGFR who were treated with EGFR TKIs. The EGFR gene copy number was assessed by silver in situ hybridization (SISH). We evaluated the clinical factors and EGFR gene copy numbers that are associated with a favorable clinical response to EGFR TKIs.

Results: The objective response rate was $12.1 \%$, while the disease control rate was $40.9 \%$. EGFR SISH analysis was feasible in 23 cases. Twelve patients tested EGFR SISH-positive, and 11 were EGFR SISH-negative, with no significant difference in tumor response and survival between EGFR SISH-positive and -negative patients. The overall median progression-free survival (PFS) and overall survival (OS) of 66 patients were 2.1 months and 9.7 months, respectively. Female sex and Eastern Cooperative Oncology Group (ECOG) performance status (PS) of 0-1 were independent predictors of PFS. ECOG PS 0-1 and a low tumor burden of extrathoracic metastasis were independent predictors of good OS.

Conclusion: Factors such as good PS, female sex, and low tumor burden may predict favorable outcomes following EGFR TKI therapy in patients with EGFR wild-type lung adenocarcinoma. However, EGFR gene copy number was not predictive of survival.

Keywords: Adenocarcinoma; Receptor, Epidermal Growth Factor; Lung Neoplasms

Address for correspondence: Cheol Hyeon Kim, M.D., Ph.D.

Division of Pulmonology, Department of Internal Medicine, Korea Cancer Center Hospital, Korea Institute of Radiological and Medical Sciences, 75

Nowon-ro, Nowon-gu, Seoul 01812, Korea

Phone: 82-2-970-1209, Fax: 82-2-970-2438, E-mail: cheol@kcch.re.kr

*Seo Yun Kim and Jae Kyung Myung contributed equally to this work.

Received: Jan. 9, 2018, Revised: Feb. 19, 2018, Accepted: Mar. 24, 2018, Published online: Jun. 19, 2018

(c) It is identical to the Creative Commons Attribution Non-Commercial License (http://creativecommons.org/licenses/by-nc/4.0/). 


\section{Introduction}

Lung cancer is a leading cause of cancer-related death worldwide ${ }^{1}$. Approximately $85 \%$ of lung cancer cases are nonsmall cell lung cancer (NSCLC). The majority of patients are diagnosed with advanced disease; outcomes remain poor, with a median survival time of $8-10$ months and a 2-year survival rate of $10 \%-20 \%^{2,3}$.

Recent advances in understanding the molecular biology of lung cancer have improved treatment of NSCLC through the development of targeted agents against molecular subgroups with specific genomic aberrations. Epidermal growth factor receptor (EGFR) has been one of the most important targets in the era of individualized therapy for NSCLC, leading to improvements in survival outcomes with a median survival of 24-30 months and an improved quality of life for patients with tumors harboring EGFR mutations, which are predominantly found in pulmonary adenocarcinomas ${ }^{4-6}$. EGFR mutations are found in approximately $10 \%$ of Caucasian patients with NSCLC and in $40 \%-50 \%$ of East Asian patients ${ }^{7}$. As for patients whose tumors have wild-type $E G F R$, only a subgroup achieves a favorable clinical response following EGFR tyrosine kinase inhibitor (TKI) treatment, suggesting that other factors may be involved. Thus, several biomarkers have been investigated in EGFR wild-type NSCLC. EGFR expression, EGFR amplification, EGFR ligand expression, and the involvement of other biomarkers that activate the EGFR signaling pathway are all associated with TKI sensitivity ${ }^{8}$. However, to date, there is no biomarker that clearly and reproducibly identifies patients with $E G F R$ wild-type NSCLC who are likely to benefit from EGFR TKIs.

Recent studies have shown that a high EGFR gene copy number is associated with a better response to TKI therapy and improved survival ${ }^{9-12}$. An increase in EGFR copy number may dysregulate the activation of the EGFR tyrosine kinase receptor protein and trigger downstream oncogenic pathways ${ }^{13}$. The gold standard for detecting EGFR gene copy number is fluorescence in situ hybridization (FISH) ${ }^{14}$. However, the disadvantages of FISH are that it is labor-intensive and timeconsuming, requires expensive equipment, and experiences a rapid loss of signal upon exposure to light. Silver-enhanced in situ hybridization (SISH) enables pathologists to evaluate slides using bright field microscopy with fully automated analysis in a cost-effective way ${ }^{15}$. Several studies reported a high concordance rate between SISH and FISH in terms of detection of EGFR amplification in NSCLC ${ }^{16}$ and glioblastoma ${ }^{17}$.

Sensitivity to EGFR TKI is associated with female sex, adenocarcinoma histology, no history of smoking, and an Asian background. However, such clinical factors are in fact predictors of EGFR mutations ${ }^{4}$. Therefore, there is insufficient information with which to identify patients with EGFR wild-type lung adenocarcinoma who would be eligible for TKI treatment.
This study aimed to determine whether an increased $E G F R$ gene copy number, as evaluated by SISH, could be used to select patients with $E G F R$ wild-type lung adenocarcinoma who are likely to benefit from EGFR TKI treatment. Furthermore, we explored clinical factors that influence the efficacy of EGFR TKIs in order to evaluate their potential applications to patient populations whose tumors are unlikely to carry EGFR mutations.

\section{Materials and Methods}

\section{Patients}

This retrospective study included patients with histologically confirmed stage IIIb, stage IV, or recurrent NSCLC who underwent EGFR mutation testing between February 2009 and December 2013 at a Korean Cancer Center Hospital. Eightyfour patients with EGFR wild-type lung adenocarcinoma received an EGFR TKI (either gefitinib or erlotinib). Patients who had received EGFR TKI for less than 2 weeks $(n=7)$, those who did not undergo follow-up chest tomography after EGFR TKI administration $(n=8)$, or those who had other primary cancers ( $n=3)$ were excluded from the study. Ultimately, 66 patients were enrolled. Gefitinib or erlotinib was administered orally (250 mg and $150 \mathrm{mg}$, respectively) once daily until disease progression or unacceptable toxicity.

Demographic and clinical characteristics including age, sex, smoking status, stage at EGFR TKI use, Eastern Cooperative Oncology Group (ECOG) performance status (PS) at EGFR TKI administration, the EGFR TKI agent administered (gefitinib or erlotinib), the number of previous chemotherapy regimens received, and extrathoracic metastatic sites (bones, brain, adrenal gland, liver, lymph nodes, and others locations as evaluated on the TKI administration start date) were retrieved from the medical records.

The study was approved by the institutional review board of Korea Cancer Center Hospital (IRB No. K-1504-001-003) and was conducted in accordance with the Declaration of Helsinki. The patients provided written informed consent to undergo the EGFR mutation and SISH tests.

\section{Response assessment}

Tumor response was assessed according to the Response Evaluation Criteria in Solid Tumors. The patients underwent chest computed tomography scans 4-8 weeks after initiating of EGFR TKI treatment, and then every 8-12 weeks thereafter. Brain magnetic resonance imaging or radionuclide bone scanning was also performed if brain or bone metastases were suspected, respectively. Patients who had complete response, partial response, or stable disease were categorized as responders; those who had progressed were categorized as 
non-responders. Progression-free survival (PFS) and overall survival (OS) were calculated from the first date of EGFR TKI treatment to the date of disease progression and the date of death or last follow-up, respectively.

\section{EGFR genotyping}

Genomic DNA was extracted from paraffin-embedded tissues, as described previously ${ }^{18}$. In patients whose only available tissue was the cytological sample obtained at initial diagnosis, methanol-fixed cytological specimens were used to extract DNA ${ }^{19}$. EGFR mutation analysis was performed by pyrosequencing using previously described methods ${ }^{20}$. The presence of $E G F R$ mutations was determined by evaluating exons $18,19,20$, and 21 .

\section{Tissue samples for EGFR SISH}

Formalin-fixed paraffin-embedded (FFPE) tumor tissue samples and cell blocks of aspiration specimens were selected from a tissue and cytology archive of samples at the Department of Pathology at Korea Cancer Center Hospital. Hematoxylin and eosin staining was performed to assess sample adequacy and to confirm the histological diagnosis. Thirty-one tissue samples from the 66 patients were available for $E G F R$ SISH analysis.

\section{EGFR SISH analysis and evaluation of SISH signals}

Dual-color dual-hapten SISH was performed according to the EGFR dinitrophenol (DNP) probe with the chromosome 7 digoxigenin (DIG) probe and ultraVIEW SISH DNP Detection Kit/ultraVIEW RED ISH DIG Detection Kit (Ventana Medical System Inc., Tucson, AZ, USA) for EGFR and chromosome 7 quantitation. The procedure was performed on an automated Ventana Benchmark XT (Ventana/Roche; Ventana Medical System Inc.) according to the manufacturer's standard protocols. Four-micrometer-thick FFPE samples from each case were deparaffinized, denatured, and pretreated with a Trisbased reaction buffer ( $\mathrm{pH}$ 7.6) in 3 cycles of 12 minutes each at $90^{\circ} \mathrm{C}$, and were then incubated with ISH Protease (Ventana/ Roche) for 24 minutes. The DNP-labeled EGFR DNA probe and DIG-labeled chromosome 7 probe were co-denatured for 12 minutes at $80^{\circ} \mathrm{C}$; slides were hybridized for 7 hours at $44^{\circ} \mathrm{C}$ and were then washed stringently 3 times for 8 minutes at $72^{\circ} \mathrm{C}$. EGFR signals were detected using the ultraVIEW SISH DNP Detection Kit. The signal was detected as silver deposits with silver acetate, hydroquinone, and hydrogen peroxide. The chromosome 7 signals were detected using the ultraVIEW RED ISH DIG Detection Kit, and were developed as stained red dots with fast red and naphthol phosphate. The slides were finally counterstained with hematoxylin II and bluing reagent before mounting. EGFR SISH scores were de- fined according to the Colorado scoring system as score 1 (1 or 2 signals in $\geq 90 \%$ of the counted nuclei [disomy]); score 2 ( 3 signals in $10-40 \%, \geq 4$ signals in $<10 \%$ [low trisomy]); score 3 ( 3 signals in $\geq 40 \%, \geq 4$ signals in $<10 \%$ [high trisomy]); score 4 ( $\geq 4$ signals in $10 \%-40 \%$ [low polysomy]); score 5 ( $\geq 4$ signals in $\geq 40 \%$ [high polysomy]); and score 6 (amplification [EGFR gene cluster $\geq 10 \%$, EGFR/chromosome 7 ratio of $\geq 2$, and $\geq 15$ signals in $\geq 10 \%])^{14}$.

Table 1. Patient characteristics

\begin{tabular}{|c|c|}
\hline Characteristic & Value $(n=66)$ \\
\hline Age, yr & $57(34-80)$ \\
\hline \multicolumn{2}{|l|}{ Sex } \\
\hline Male & $44(66.7)$ \\
\hline Female & $22(33.3)$ \\
\hline \multicolumn{2}{|l|}{ Smoking history } \\
\hline Ever smoker & $46(69.1)$ \\
\hline Never smoker & $20(30.3)$ \\
\hline \multicolumn{2}{|l|}{ Stage at EGFR TKI use } \\
\hline IIIb & $2(3.0)$ \\
\hline IV & $64(97.0)$ \\
\hline \multicolumn{2}{|l|}{ ECOG PS at TKI treatment } \\
\hline $0-1$ & $41(62.1)$ \\
\hline$\geq 2$ & $25(37.9)$ \\
\hline \multicolumn{2}{|l|}{ TKI } \\
\hline Gefitinib & $42(63.6)$ \\
\hline Erlotinib & $24(36.4)$ \\
\hline \multicolumn{2}{|l|}{ Line } \\
\hline 1st- or 2nd-line & $36(54.5)$ \\
\hline 3rd-line or above & $30(45.5)$ \\
\hline Extrathoracic metastasis & $52(78.8)$ \\
\hline Brain & $15(22.7)$ \\
\hline Bone & $25(37.9)$ \\
\hline \multicolumn{2}{|c|}{ No. of extrathoracic metastasis sites } \\
\hline 1 & $36(54.5)$ \\
\hline 2 & $10(15.2)$ \\
\hline$\geq 3$ & $6(9.1)$ \\
\hline \multicolumn{2}{|l|}{ Tumor response } \\
\hline Partial response & $8(12.1)$ \\
\hline Stable disease & $19(28.8)$ \\
\hline Progressive disease & $39(59.1)$ \\
\hline
\end{tabular}

Values are presented as median (range) or number (\%). EGFR: epidermal growth factor receptor; TKI: tyrosine kinase inhibitor; ECOG PS: Eastern Cooperative Oncology Group performance status. 


\section{Statistical analyses}

Categorical variables were analyzed using Fisher exact test, while continuous variables were analyzed using the MannWhitney test. The associated factors with treatment response of EGFR TKI were identified by univariate and multivariate logistic regression. PFS and OS were estimated by using the Kaplan-Meier method. Comparisons between groups were performed using the log-rank test for time-to-event variables. Cox proportional hazards models were used to evaluate independent predictive factors for survival. All statistical analyses were performed using SPSS software version 18.0 (SPSS Inc., Chicago, IL, USA), and p-values of $<0.05$ were considered sta- tistically significant.

\section{Results}

\section{Patients characteristics}

A total of 66 patients who received EGFR TKIs for lung adenocarcinoma with $E G F R$ wild-type were identified for the analyses. The median follow-up time was 17.0 months (range, 1.9-81.3 months). The patients' median age was 57 years, and $66.7 \%$ were men (Table 1). The proportion of ever smokers was $69.1 \%$. Approximately half of all patients were treated with

Table 2. Association of EGFR SISH status with clinical characteristics

\begin{tabular}{|c|c|c|c|c|}
\hline Variable & All patients $(n=23)$ & SISH-positive $(n=12)$ & SISH-negative $(n=11)$ & p-value \\
\hline Age, yr & $60(34-78)$ & $59(47-75)$ & $62(34-78)$ & 0.418 \\
\hline Sex & & & & 0.640 \\
\hline Male & $17(73.9)$ & $8(66.7)$ & $9(81.8)$ & \\
\hline Female & $6(26.1)$ & $4(33.3)$ & $2(18.2)$ & \\
\hline Smoking history & & & & 0.371 \\
\hline Ever smoker & $16(69.6)$ & $7(58.3)$ & $9(81.8)$ & \\
\hline Never smoker & $7(30.4)$ & $5(41.7)$ & $2(18.2)$ & \\
\hline ECOG PS at TKI treatment & & & & 0.414 \\
\hline $0-1$ & $10(43.5)$ & $4(33.3)$ & $6(54.5)$ & \\
\hline$\geq 2$ & $13(56.5)$ & $8(66.7)$ & $5(45.5)$ & \\
\hline TKI & & & & 0.680 \\
\hline Gefitinib & $14(60.9)$ & $8(66.7)$ & $6(54.5)$ & \\
\hline Erlotinib & $9(39.1)$ & $4(33.3)$ & $5(45.5)$ & \\
\hline Line & & & & 0.680 \\
\hline 1st- or 2nd-line & $10(43.5)$ & $6(50.0)$ & $4(36.4)$ & \\
\hline 3rd-line or above & $13(56.5)$ & $6(50.0)$ & $7(63.6)$ & \\
\hline Extrathoracic metastasis & $21(91.3)$ & $11(91.7)$ & $10(90.9)$ & $>0.999$ \\
\hline Brain & $6(26.1)$ & $4(33.3)$ & $2(18.2)$ & 0.640 \\
\hline Bone & $11(47.8)$ & $5(41.7)$ & $6(54.5)$ & 0.684 \\
\hline No. of extrathoracic metastasis sites & & & & 0.107 \\
\hline 1 & $13(56.5)$ & $9(75.0)$ & $4(36.4)$ & \\
\hline 2 & $5(21.7)$ & $1(8.3)$ & $4(36.4)$ & \\
\hline$\geq 3$ & $3(13.0)$ & $1(8.3)$ & $2(18.2)$ & \\
\hline \multicolumn{5}{|l|}{ Tumor response } \\
\hline Partial response & $2(8.7)$ & $2(16.7)$ & $0(0)$ & 0.478 \\
\hline Stable disease & $4(17.4)$ & $2(16.7)$ & $2(18.2)$ & $>0.999$ \\
\hline Progressive disease & $17(73.9)$ & $8(66.7)$ & $9(81.8)$ & 0.640 \\
\hline
\end{tabular}

Values are presented as median (range) or number (\%).

EGFR: epidermal growth factor receptor; SISH: silver in situ hybridization; ECOG PS: Eastern Cooperative Oncology Group performance status; TKI: tyrosine kinase inhibitor. 
EGFR TKIs beyond second-line therapy. Most patients (97.0\%) had stage IV disease; $78.8 \%$ had extrathoracic metastasis.

The tumor responses to EGFR TKIs are shown in Table 1; the objective response rate was $12.1 \%$ while the disease control rate was $40.9 \%$.

\section{EGFR SISH and TKI efficacy}

We performed EGFR SISH in 31 NSCLC specimens; analysis was possible in 23 of these cases. Disomy was present in one patient $(4.3 \%)$, low trisomy in three (13.0\%), high trisomy in three (13.0\%), low polysomy in five (21.7\%), high polysomy in $10(43.5 \%)$, and gene amplification in one (4.3\%). SISH positivity was defined as high polysomy or gene amplification. Twelve patients (52.2\%) were categorized as EGFR SISH positive (high $E G F R$ copy number), and 11 (47.8\%) were characterized as EGFR SISH negative (low EGFR copy number). There was no significant difference in clinical characteristics between EGFR SISH-positive and -negative patients (Table
2). There was a trend toward higher response and disease control rates in SISH-positive patients compared to SISHnegative patients ( $16.7 \%$ vs. $0 \%, \mathrm{p}=0.478$ and $33.4 \%$ vs. $18.2 \%$, $\mathrm{p}=0.640$, respectively). The median PFS and OS were longer in SISH-positive patients than in SISH-negative patients (PFS: 2.0 months, vs. 1.0 month respectively; hazard ratio [HR] $0.57 ; 95 \%$ confidence interval [CI], $0.24-1.35 ; \mathrm{p}=0.201$ and OS: 7.5 months vs. 3.0 months, respectively; $\mathrm{HR}, 0.55$; 95\% CI, $0.23-1.30$; $\mathrm{p}=0.173$ ), but the differences were not statistically significant.

To investigate the baseline clinical variables that could predict treatment response to EGFR TKIs, logistic regression models were used to evaluate the correlation between these factors and treatment response. On univariate analyses (Table 3 ), female sex and never smoker were significantly associated with better treatment response (odds ratio [OR], 3.10; 95\% CI, 1.07-8.94; $\mathrm{p}=0.037$ and OR, 3.10; 95\% CI, 1.05-9.19; $\mathrm{p}=0.041$ ). But, there was no clinical factor to predict treatment response on multivariate analysis.

Table 3. Analysis of clinical variables associated with treatment response of EGFR TKI

\begin{tabular}{|c|c|c|c|c|}
\hline \multirow{2}{*}{ Predictor } & \multicolumn{2}{|c|}{ Univariate } & \multicolumn{2}{|c|}{ Multivariate } \\
\hline & OR $(95 \% \mathrm{CI})$ & p-value & OR $(95 \% \mathrm{CI})$ & p-value \\
\hline \multicolumn{5}{|l|}{ Age, yr } \\
\hline$\geq 60$ vs. $<60$ & $2.04(0.72-5.75)$ & 0.180 & $2.11(0.71-6.27)$ & 0.177 \\
\hline \multicolumn{5}{|l|}{ Sex } \\
\hline Male vs. Female & $3.10(1.07-8.94)$ & 0.037 & $1.83(0.33-10.18)$ & 0.492 \\
\hline \multicolumn{5}{|l|}{ Smoking history } \\
\hline Ever vs. Never & $3.10(1.05-9.19)$ & 0.041 & $2.00(0.34-11.74)$ & 0.443 \\
\hline \multicolumn{5}{|l|}{ Stage at EGFR TKIs use } \\
\hline IV vs. IIIb & $1.46(0.09-24.43)$ & 0.792 & - & - \\
\hline \multicolumn{5}{|l|}{ ECOG PS at TKI treatment } \\
\hline$\geq 2$ vs. $0-1$ & $2.45(0.84-7.11)$ & 0.100 & - & - \\
\hline \multicolumn{5}{|l|}{ TKIs } \\
\hline Gefitinib vs. Erlotinib & $1.25(0.45-3.49)$ & 0.670 & - & - \\
\hline \multicolumn{5}{|l|}{ Line } \\
\hline 3rd-line and above vs. 1st- or 2nd-line & $0.72(0.27-1.95)$ & 0.523 & - & - \\
\hline Extrathoracic metastasis & $0.63(0.19-2.05)$ & 0.438 & - & - \\
\hline Brain & $1.36(0.43-4.33)$ & 0.607 & - & - \\
\hline Bone & $1.23(0.45-3.37)$ & 0.690 & - & - \\
\hline \multicolumn{5}{|l|}{ No. of extrathoracic metastasis sites } \\
\hline$\geq 3$ vs. $1-2$ & $1.43(0.24-8.41)$ & 0.693 & - & - \\
\hline \multicolumn{5}{|l|}{ SISH } \\
\hline Negative vs. Positive & $2.25(0.32-15.76)$ & 0.414 & - & - \\
\hline
\end{tabular}

EGFR: epidermal growth factor receptor; TKI: tyrosine kinase inhibitor; OR: odds ratio; CI: confidence interval; ECOG PS: Eastern Cooperative Oncology Group performance status; SISH: silver in situ hybridization. 
Table 4. Analysis of clinical variables associated with progression-free survival

\begin{tabular}{|c|c|c|c|c|}
\hline \multirow{2}{*}{ Predictor } & \multicolumn{2}{|c|}{ Univariate } & \multicolumn{2}{|c|}{ Multivariate } \\
\hline & HR (95\% CI) & p-value & HR (95\% CI) & p-value \\
\hline \multicolumn{5}{|l|}{ Age, yr } \\
\hline$\geq 60$ vs. $<60$ & $0.86(0.51-1.46)$ & 0.573 & $1.03(0.60-1.77)$ & 0.924 \\
\hline \multicolumn{5}{|l|}{ Sex } \\
\hline Male vs. Female & $0.52(0.29-0.92)$ & 0.024 & $0.48(0.27-0.86)$ & 0.013 \\
\hline \multicolumn{5}{|l|}{ Smoking history } \\
\hline Ever vs. Never & $0.57(0.32-1.02)$ & 0.057 & - & - \\
\hline \multicolumn{5}{|l|}{ Stage at EGFR TKIs use } \\
\hline IV vs. IIIb & $1.08(0.56-1.65)$ & 0.876 & - & - \\
\hline \multicolumn{5}{|l|}{ ECOG PS at TKI treatment } \\
\hline$\geq 2$ vs. $0-1$ & $0.50(0.30-0.85)$ & 0.011 & $0.47(0.27-0.81)$ & 0.006 \\
\hline \multicolumn{5}{|l|}{ TKIs } \\
\hline Gefitinib vs. Erlotinib & $1.05(0.61-1.81)$ & 0.869 & - & - \\
\hline \multicolumn{5}{|l|}{ Line } \\
\hline 1st- or 2nd-line vs. 3rd-line and above & $1.11(0.66-1.87)$ & 0.695 & - & - \\
\hline Extrathoracic metastasis & $1.17(0.61-2.23)$ & 0.636 & - & - \\
\hline Brain & $0.64(0.33-1.22)$ & 0.170 & - & - \\
\hline Bone & $0.985(0.57-1.69)$ & 0.955 & - & - \\
\hline \multicolumn{5}{|l|}{ No. of extrathoracic metastasis sites } \\
\hline$\geq 3$ vs. $1-2$ & $0.47(0.19-1.15)$ & 0.099 & - & - \\
\hline \multicolumn{5}{|l|}{ SISH } \\
\hline Negative vs. Positive & $0.54(0.22-1.28)$ & 0.158 & - & - \\
\hline
\end{tabular}

HR: hazard ratio; CI: confidence interval; EGFR: epidermal growth factor receptor; TKI: tyrosine kinase inhibitor; ECOG PS: Eastern Cooperative Oncology Group performance status; SISH: silver in situ hybridization.

\section{Survival outcomes}

The overall median PFS and OS rates of all 66 patients were 2.1 months (95\% CI, 1.77-2.43) and 9.7 months (95\% CI, 6.3513.05), respectively. Univariate analysis of PFS showed that female sex and ECOG PS scores of 0-1 had significantly positive effects on PFS; both factors were also significant independent predictors of PFS on multivariate analysis (Table 4). Furthermore, female sex, never smoker status, ECOG PS 0-1, and a low number of extrathoracic metastasis sites were significant predictors of longer OS on univariate analysis (Table 5). Treatment with EGFR TKIs beyond second-line therapy was associated with poor OS. On multivariate analysis, an ECOG PS 0-1 and a low tumor burden of extrathoracic metastases were independent predictors of longer OS.

\section{Discussion}

In our study of 66 patients with EGFR wild-type lung ad- enocarcinoma who received EGFR TKIs, the response and disease control rates were $12.1 \%$ and $40.9 \%$, respectively, which are similar to results from previous studies. The FirstSIGNAL trial reported a $25.9 \%$ response rate and a $40.7 \%$ disease control rate to gefitinib in never smokers with $E G F R$ wildtype lung adenocarcinoma ${ }^{21}$. Another Asian study reported a $21.9 \%$ response rate and a $38.4 \%$ disease control rate with $\mathrm{EGFR} \mathrm{TKI}^{22}$. However, the response rate in patients with $E G F R$ wild-type was only $1.1 \%$ in the Iressa Pan-Asia Study (IPASS) ${ }^{4}$. These discrepant results may be explained by differences in the EGFR mutation detection method. Pyrosequencing was used in our study, whereas the more sensitive amplificationrefractory mutation system was used in the IPASS trial; this might account for a relatively higher false-negative rate.

The role of EGFR copy number as a predictive biomarker for the efficacy of EGFR TKIs remains controversial ${ }^{23}$. Several studies suggested that a high EGFR gene copy number correlated with EGFR TKI treatment response and longer survival in patients with $E G F R$ wild-type lung cancer $^{9-12}$. In a recent study of patients with EGFR wild-type NSCLC, those who 
Table 5. Analysis of clinical variables associated with overall survival

\begin{tabular}{|c|c|c|c|c|}
\hline \multirow{2}{*}{ Predictor } & \multicolumn{2}{|c|}{ Univariate } & \multicolumn{2}{|c|}{ Multivariate } \\
\hline & HR (95\% CI) & p-value & HR (95\% CI) & p-value \\
\hline \multicolumn{5}{|l|}{ Age, yr } \\
\hline$\geq 60$ vs. $<60$ & $0.62(0.36-1.05)$ & 0.077 & $0.69(0.37-1.31)$ & 0.260 \\
\hline \multicolumn{5}{|l|}{ Sex } \\
\hline Male vs. Female & $0.39(0.22-0.70)$ & 0.002 & $0.50(0.09-2.89)$ & 0.436 \\
\hline \multicolumn{5}{|l|}{ Smoking history } \\
\hline Ever vs. Never & $0.47(0.26-0.85)$ & 0.012 & $0.72(0.12-4.38)$ & 0.717 \\
\hline \multicolumn{5}{|l|}{ Stage at EGFR TKI use } \\
\hline IV vs. IIIlb & $0.87(0.21-3.60)$ & 0.846 & - & - \\
\hline \multicolumn{5}{|l|}{ ECOG PS at TKI treatment } \\
\hline$\geq 2$ vs. $0-1$ & $0.30(0.17-0.53)$ & $<0.001$ & $0.34(0.17-0.65)$ & 0.001 \\
\hline \multicolumn{5}{|l|}{ TKIs } \\
\hline Gefitinib vs. Erlotinib & $1.44(0.82-2.53)$ & 0.210 & - & - \\
\hline \multicolumn{5}{|l|}{ Line } \\
\hline 1st- or 2nd-line vs. 3rd-line and above & $1.72(1.01-2.93)$ & 0.046 & $1.07(0.57-2.03)$ & 0.831 \\
\hline Extrathoracic metastasis & $1.83(0.95-3.50)$ & 0.070 & - & - \\
\hline Brain & $0.93(0.49-1.76)$ & 0.823 & - & - \\
\hline Bone & $1.25(0.73-2.16)$ & 0.423 & - & - \\
\hline \multicolumn{5}{|l|}{ No. of extrathoracic metastasis sites } \\
\hline$\geq 3$ vs. $1-2$ & $0.25(0.09-0.64)$ & 0.004 & $0.29(0.11-0.77)$ & 0.013 \\
\hline \multicolumn{5}{|l|}{ SISH } \\
\hline Negative vs. Positive & $0.55(0.23-1.30)$ & 0.173 & - & - \\
\hline
\end{tabular}

HR: hazard ratio; CI: confidence interval; EGFR: epidermal growth factor receptor; TKI: tyrosine kinase inhibitor; ECOG PS: Eastern Cooperative Oncology Group performance status; SISH: silver in situ hybridization.

were EGFR FISH-positive achieved a significantly higher response rate $(17.7 \%$ vs. $8.6 \%$, $\mathrm{p}=0.047)$ and disease control rate (67.7\% vs. $35.7 \%$, $\mathrm{p}<0.001)$, as well as longer PFS (4.4 months vs. 2.0 months; HR, 0.56; 95\% CI, 0.41-0.75; $\mathrm{p}<0.001)$ and OS (25.0 months vs. 14.2 months; HR, 0.60; 95\% CI, 0.41-0.89; $\mathrm{p}=0.010$ ) than patients who were FISH-negative .

We showed that EGFR SISH was positive in 12 of 23 patients (52.2\%) with EGFR wild-type lung adenocarcinoma. PFS and OS tended to be longer in SISH-positive patients than SISH negative patients. Moreover, the response and disease control rates were higher in SISH-positive patients than in SISH-negative patients. However, there were no significant differences in survival and tumor response between the EGFR SISH-positive and -negative patients. Lee et al. ${ }^{24}$ showed that a high EGFR gene copy number was associated with better responses to EGFR TKI ( $27.3 \%$ vs. $4.2 \%$, $\mathrm{p}=0.082)$ and PFS ( 4.10 months [95\% CI, 1.66-6.54] vs. 2.10 months [95\% CI, 1.21$2.99], \mathrm{p}=0.201$ ) in patients with $E G F R$ wild-type squamous cell carcinoma; however, these associations did not reach statistical significance. Li et al. ${ }^{25}$ investigated the efficacy of erlotinib versus pemetrexed as second-line treatments for patients with $E G F R$ wild-type and EGFR FISH-positive lung adenocarcinoma, and found that the median PFS was 4.1 months (95\% CI, 1.6-6.6) and 3.9 months (95\% CI, 2.7-5.1), respectively, in the erlotinib and pemetrexed arms (HR, 0.92; 95\% CI, 0.62-1.37; $\mathrm{p}=0.693$ ). The objective response rate appeared to be higher among patients receiving erlotinib compared with those receiving pemetrexed (19.7\% vs. $8.1 \%$, respectively; $\mathrm{p}=0.062$ ). Their study did not reveal differences in survival and responses between erlotinib and pemetrexed in patients with EGFR wild-type and EGFR FISH-positive adenocarcinoma.

In our study, good PS and female sex were associated with better PFS. An ECOG PS 0-1 and a low tumor burden of extrathoracic metastases were independent predictors of longer OS. Several studies reported that PS was predictive factor for response to EGFR TKI in patients with wild-type $\mathrm{EGFR}^{26,27}$ and female sex was independent good predictors of gefitinib treatment in patients with advanced non-small cell lung cancer ${ }^{28,29}$.

Sanchez de Cos Escuin et al. ${ }^{30}$ reported that the number of metastatic sites and of lesions in patients with isolated metas- 
tasis had prognostic relevance. Tumor burden is a predictive factor for response and survival in patients with NSCLC irrespective of the therapeutic agents employed, including cytotoxic chemotherapy and TKI ${ }^{31-33}$. Higher tumor burdens may reflect the rapid growth and greater number of cancer cells and be associated with poor prognosis.

The present study has several limitations. It was underpowered due to the small sample size, which may have been a factor in the failure to detect differences between EGFR SISHpositive and -negative patients. As ours was a single-center retrospective study, future prospective studies ought to be conducted to validate our findings.

In conclusion, we found that PS, female sex, and tumor burden are predictive factors of the clinical benefit of EGFR TKI therapy in $E G F R$ wild-type lung adenocarcinoma. However, EGFR SISH status was not predictive of EGFR TKI efficacy.

\section{Authors' Contributions}

Conceptualization: Kim CH, Kim SY. Formal analysis: Kim SY, Myung JK. Data curation: all authors. Writing - original draft preparation: Kim SY, Myung JK. Writing - review and editing: Kim SY, Myung JK. Approval of final manuscript: all authors.

\section{Conflicts of Interest}

No potential conflict of interest relevant to this article was reported.

\section{Acknowledgments}

This study was supported by a grant of the Korea Institute of Radiological and Medical Sciences, funded by Ministry of Science, ICT and Future Planning, Republic of Korea (1711045543/50474-2017). The authors have no financial conflicts of interest.

\section{References}

1. Torre LA, Siegel RL, Jemal A. Lung cancer statistics. Adv Exp Med Biol 2016;893:1-19.

2. Schiller JH, Harrington D, Belani CP, Langer C, Sandler A, Krook J, et al. Comparison of four chemotherapy regimens for advanced non-small-cell lung cancer. N Engl J Med 2002;346:92-8.

3. Fossella F, Pereira JR, von Pawel J, Pluzanska A, Gorbounova V, Kaukel E, et al. Randomized, multinational, phase III study of docetaxel plus platinum combinations versus vinorelbine plus cisplatin for advanced non-small-cell lung cancer: the TAX 326 study group. J Clin Oncol 2003;21:3016-24.

4. Mok TS, Wu YL, Thongprasert S, Yang CH, Chu DT, Saijo N, et al. Gefitinib or carboplatin-paclitaxel in pulmonary adenocarcinoma. N Engl J Med 2009;361:947-57.

5. Maemondo M, Inoue A, Kobayashi K, Sugawara S, Oizumi S, Isobe $\mathrm{H}$, et al. Gefitinib or chemotherapy for non-small-cell lung cancer with mutated EGFR. N Engl J Med 2010;362:23808.

6. Rosell R, Carcereny E, Gervais R, Vergnenegre A, Massuti B, Felip E, et al. Erlotinib versus standard chemotherapy as firstline treatment for European patients with advanced EGFR mutation-positive non-small-cell lung cancer (EURTAC): a multicentre, open-label, randomised phase 3 trial. Lancet Oncol 2012;13:239-46.

7. Hirsch FR, Bunn PA Jr. EGFR testing in lung cancer is ready for prime time. Lancet Oncol 2009;10:432-3.

8. Laurie SA, Goss GD. Role of epidermal growth factor receptor inhibitors in epidermal growth factor receptor wild-type nonsmall-cell lung cancer. J Clin Oncol 2013;31:1061-9.

9. Wang F, Fu S, Shao Q, Zhou YB, Zhang X, Zhang X, et al. High EGFR copy number predicts benefits from tyrosine kinase inhibitor treatment for non-small cell lung cancer patients with wild-type EGFR. J Transl Med 2013;11:90.

10. Hou MM, Huang SF, Kuo HP, Yang CT, Tsai YH, Yu CT, et al. Erlotinib treatment in patients with advanced lung adenocarcinoma with CISH-positive and CISH-negative EGFR gene alterations. Anticancer Res 2012;32:1107-12.

11. Hirsch FR, Varella-Garcia M, McCoy J, West H, Xavier AC, Gumerlock P, et al. Increased epidermal growth factor receptor gene copy number detected by fluorescence in situ hybridization associates with increased sensitivity to gefitinib in patients with bronchioloalveolar carcinoma subtypes: a Southwest Oncology Group Study. J Clin Oncol 2005;23:6838-45.

12. Cappuzzo F, Hirsch FR, Rossi E, Bartolini S, Ceresoli GL, Bemis L, et al. Epidermal growth factor receptor gene and protein and gefitinib sensitivity in non-small-cell lung cancer. J Natl Cancer Inst 2005;97:643-55.

13. Olayioye MA, Neve RM, Lane HA, Hynes NE. The ErbB signaling network: receptor heterodimerization in development and cancer. EMBO J 2000;19:3159-67.

14. Varella-Garcia M, Diebold J, Eberhard DA, Geenen K, Hirschmann A, Kockx M, et al. EGFR fluorescence in situ hybridisation assay: guidelines for application to non-small-cell lung cancer. J Clin Pathol 2009;62:970-7.

15. Fritzsche FR, Bode PK, Moch H, Kristiansen G, Varga Z, Bode B. Determination of the Her-2/neu gene amplification status in cytologic breast cancer specimens using automated silverenhanced in-situ hybridization (SISH). Am J Surg Pathol 2010;34:1180-5.

16. Wulf MA, Bode B, Zimmermann D, Rufibach K, Weder W, Moch $\mathrm{H}$, et al. Silver-enhanced in situ hybridization for determination of EGFR copy number alterations in non-small cell 
lung cancer. Am J Surg Pathol 2012;36:1801-8.

17. Gaiser T, Waha A, Moessler F, Bruckner T, Pietsch T, von Deimling A. Comparison of automated silver enhanced in situ hybridization and fluorescence in situ hybridization for evaluation of epidermal growth factor receptor status in human glioblastomas. Mod Pathol 2009;22:1263-71.

18. Na II, Kang HJ, Cho SY, Koh JS, Lee JK, Lee BC, et al. EGFR mutations and human papillomavirus in squamous cell carcinoma of tongue and tonsil. Eur J Cancer 2007;43:520-6.

19. Boldrini L, Gisfredi S, Ursino S, Camacci T, Baldini E, Melfi $\mathrm{F}$, et al. Mutational analysis in cytological specimens of advanced lung adenocarcinoma: a sensitive method for molecular diagnosis. J Thorac Oncol 2007;2:1086-90.

20. Na II, Park JH, Choe du H, Lee JK, Koh JS. Association of epidermal growth factor receptor mutations with metastatic presentations in non-small cell lung cancer. ISRN Oncol 2011;2011:756265.

21. Han JY, Park K, Kim SW, Lee DH, Kim HY, Kim HT, et al. FirstSIGNAL: first-line single-agent iressa versus gemcitabine and cisplatin trial in never-smokers with adenocarcinoma of the lung. J Clin Oncol 2012;30:1122-8.

22. Chang MH, Ahn HK, Lee J, Jung CK, Choi YL, Park YH, et al. Clinical impact of amphiregulin expression in patients with epidermal growth factor receptor ( $E G F R$ ) wild-type nonsmall cell lung cancer treated with EGFR-tyrosine kinase inhibitors. Cancer 2011;117:143-51.

23. Dahabreh IJ, Linardou H, Kosmidis P, Bafaloukos D, Murray S. EGFR gene copy number as a predictive biomarker for patients receiving tyrosine kinase inhibitor treatment: a systematic review and meta-analysis in non-small-cell lung cancer. Ann Oncol 2011;22:545-52.

24. Lee Y, Shim HS, Park MS, Kim JH, Ha SJ, Kim SH, et al. High $E G F R$ gene copy number and skin rash as predictive markers for EGFR tyrosine kinase inhibitors in patients with advanced squamous cell lung carcinoma. Clin Cancer Res 2012;18:1760-8.

25. Li N, Ou W, Yang H, Liu QW, Zhang SL, Wang BX, et al. A randomized phase 2 trial of erlotinib versus pemetrexed as second-line therapy in the treatment of patients with advanced
EGFR wild-type and EGFR FISH-positive lung adenocarcinoma. Cancer 2014;120:1379-86.

26. Ren S, Su C, Wang Z, Li J, Fan L, Li B, et al. Epithelial phenotype as a predictive marker for response to EGFR-TKIs in non-small cell lung cancer patients with wild-type EGFR. Int J Cancer 2014;135:2962-71.

27. Lai SW, Ho CL, Dai MS, Chen WL, Chang PY, Wu YY, et al. Gefitinib as first-line treatment for patients with epidermal growth factor receptor-mutated advanced lung adenocarcinoma: a single institution experience in Taiwan. J BUON 2014;19:459-65.

28. Kaneda H, Tamura K, Kurata T, Uejima H, Nakagawa K, Fukuoka M. Retrospective analysis of the predictive factors associated with the response and survival benefit of gefitinib in patients with advanced non-small-cell lung cancer. Lung Cancer 2004;46:247-54.

29. Yang CH, Shih JY, Chen KC, Yu CJ, Yang TY, Lin CP, et al. Survival outcome and predictors of gefitinib antitumor activity in East Asian chemonaive patients with advanced nonsmall cell lung cancer. Cancer 2006;107:1873-82.

30. Sanchez de Cos Escuin J, Abal Arca J, Melchor Iniguez R, Miravet Sorribes L, Nunez Ares A, Hernandez Hernandez JR, et al. Tumor, node and metastasis classification of lung cancer: Mla versus Mlb: analysis of $\mathrm{M}$ descriptors and other prognostic factors. Lung Cancer 2014;84:182-9.

31. Park S, Kim HJ, Choi CM, Lee DH, Kim SW, Lee JS, et al. Predictive factors for a long-term response duration in nonsquamous cell lung cancer patients treated with pemetrexed. BMC Cancer 2016;16:417.

32. Park JH, Kim TM, Keam B, Jeon YK, Lee SH, Kim DW, et al. Tumor burden is predictive of survival in patients with nonsmall-cell lung cancer and with activating epidermal growth factor receptor mutations who receive gefitinib. Clin Lung Cancer 2013;14:383-9.

33. Oh Y, Taylor S, Bekele BN, Debnam JM, Allen PK, Suki D, et al. Number of metastatic sites is a strong predictor of survival in patients with nonsmall cell lung cancer with or without brain metastases. Cancer 2009;115:2930-8. 